\title{
Justification of the Calculation Method for Arch Support with Rock Grouting
}

\author{
Victor Tatsienko ${ }^{1,}$, , Vyacheslav Gogolin ${ }^{1}$, Inna Ermakova ${ }^{1}$, and Alexander Liskovets ${ }^{1}$ \\ ${ }^{1}$ T.F. Gorbachev Kuzbass State Technical University, 650000, Kemerovo, Russian Federation
}

\begin{abstract}
This paper proposes a new method for calculating arch support with grouted space behind the support. The analysis of existing installations and methods for the calculation of frame supports was made. It has been established that the existing methods of frame parts calculation do not take into account the presence of grouted space behind the support. It is proposed to take into account the presence of the grouted layer in the space behind the support when it interacts with the rock mass. The formation of partially disturbed rock adjacent to the grouted layer is taken into account in the behaviour of rocks. In this method, the arch support is replaced with a ring support. The finite element method establishes the reduced dimensions of the ring support and its module of linear deformations, corresponding to these values of the arch support when its bearing capacity is lost. The scheme for calculating arch support ultimately boils down to considering the interaction of the support, the grouted layer, the zone of partially destroyed rocks and the rest of the mass of intact rocks in the hydrostatic field of rock pressure.
\end{abstract}

\section{Introduction}

The task of calculating the support for development and capital workings is one of the main tasks of rock mechanics. To solve this task, experimental and theoretical approaches were used.

The results of experimental studies are presented in numerous works, summarized in monographs [1, 2], in guidelines for performing design work, and also in textbooks. Performing a complete review of these studies is a great independent task. The obtained calculation formulas for the support load, the support displacement and its bearing capacity are applicable only in those mining and geological conditions in which experimental measurements were carried out. The desire to take into account all possible factors affecting the state of the support results in appearance of up to ten empirical coefficients. A fairly approximate definition of empirical coefficients significantly reduces the accuracy of the design formulas. So, the less empirical coefficients are used, the more accurate the formulas are, but in a narrower area of their application

The theoretical approach allows us to obtain more general laws of the geomechanical state of the support and rock mass and to clarify empirical formulas. One of the directions of theoretical calculations of support is based on knowledge of the anticipated loads on the

${ }^{*}$ Corresponding author: ipeb@,kuzstu.ru 
support and further use of computational methods of material resistamce and structural mechanics. However, the values of the loads on the support can be found only after solving the problem of the interaction of support with the rock strata. Such an approach has been implemented in a number of works in which different types of supports were studied.

A theoretical assessment of the effect of the grouted behind-the-support space on the stress-strain state of the rock strata was made in the study [3-9]. However, the pressure on the support was preliminary set as the working resistance of the support, which is a separate task.

\section{Materials and technique}

In this paper, we consider the formulation of the problem of calculating the stress-strain state of the system 'frame support - grouted layer - rock mass'.

The general calculation scheme is presented in Fig. 1.

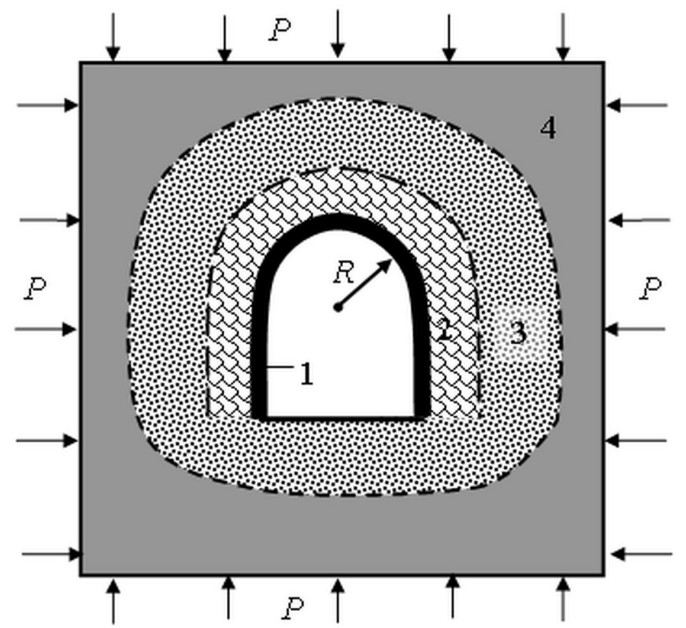

Fig. 1. Calculation scheme: 1 - support; 2 - grouted layer; 3 - partly broken ground; 4 - host rock.

The strain-stress state of the region of computation is considered when the support crown interacts with the grouted layer and the rock strata. The calculation is carried out for a circular roadway with a radius $R$ equal to the radius of the roof crown. If the sectional area of the roadway $S$ is known, then the reduced radius $R=\sqrt{S / \pi}$ is considered. The calculation area consists of four blocks.

Block 1 is a support with deformation and strength characteristics: $E_{k}$ - Young modulus; $v_{k}$ is the coefficient of transverse deformations; $\sigma_{k}$ - tensile strength of the lining material (steel).

Block 2 is a layer of cementing stone with deformation and strength characteristics: $E_{t}-$ Young modulus; $v_{t}$ - Poisson ratio; $\sigma_{t}$-compressive strength of cement stone.

Block 3 is a zone of partially destroyed rock (over-limit deformation), in which circumferential and radial stresses are proportional.

Block 4 is a zone of host rock that is in an elastic state with deformation and strength characteristics: $E$ - Young modulus of rock; $v$ - Poisson ratio; $\sigma_{s}$ - tensile strength.

The boundaries of the zones maintain continuity, therefore, at the crossing of the boundaries of the zones, the radial displacements and stresses should be continuous. 
The equilibrium equation in stresses for an axisymmetric problem has the following view [10]:

$$
\frac{d \sigma_{r}}{d r}+\frac{\sigma_{r}-\sigma_{\theta}}{r}=0
$$

where, $r$ - the distance from the centre of the roadway;

$\sigma_{r}, \sigma \theta-$ radial and circumferential stress.

This equation is applied to each block of the calculation area.

The internal and external pressure levels $p_{1}, p_{2}$ are assigned to the block, and the boundary conditions have the following view

$$
\left\{\begin{array}{c}
\sigma_{r}\left(R_{1}\right)=p_{1} \\
\sigma_{r}\left(R_{2}\right)=p_{2} .
\end{array}\right.
$$

Here, $R_{1}, R_{2}$ - internal and external radii of the ring zone.

The common solution to this equation for the elastic (lineary elastic) behaviour of the support material and rock strata has the following view [10]:

$$
\left\{\begin{array}{l}
\sigma_{r}(r)=\frac{p_{2} \cdot R_{2}^{2}-p_{1} \cdot R_{1}^{2}}{R_{2}^{2}-R_{1}^{2}}-\frac{R_{2}^{2} \cdot R_{1}^{2} \cdot\left(p_{2}-p_{1}\right)}{R_{2}^{2}-R_{1}^{2}} \cdot \frac{1}{r^{2}} \\
\sigma_{\theta}(r)=\frac{p_{2} \cdot R_{2}^{2}-p_{1} \cdot R_{1}^{2}}{R_{2}^{2}-R_{1}^{2}}+\frac{R_{2}^{2} \cdot R_{1}^{2} \cdot\left(p_{2}-p_{1}\right)}{R_{2}^{2}-R_{1}^{2}} \cdot \frac{1}{r^{2}},
\end{array}\right.
$$

where, $r$ - the distance from the centre of the roadway.

At the internal border of the area from the roadway side there is no presuure on the support: $\sigma_{r}(R)=0$.

At the remote distance from the roadway, the distribution of stresses in the rock strata is taken as hydrostatic: $\sigma_{r}(\infty)=\sigma_{\theta}(\infty)=p$. In particular, $p=\gamma H$, where $\gamma$ is the specific weight of the rock, $H$ is the depth of roadway.

Let us consider a ring support with an inner radius $\mathrm{R}$ and outer radius $R_{1}$. The height of the support is $R_{1}-R=h_{k}$. The inner surface of the support is not loaded, the outer surface is under pressure from the grouting $q$. Therefore, the boundary conditions have the following view:

$$
\left\{\begin{array}{c}
\sigma_{r}(R)=0 ; \\
\sigma_{r}\left(R_{1}\right)=q .
\end{array}\right.
$$

In accordance with the general solution (3) and with consideration of the boundary conditions (4), the obtained stress distribution in the support is as follows:

$$
\left\{\begin{array}{l}
\sigma_{r}(r)=\frac{q \cdot R_{1}^{2}}{R_{1}^{2}-R^{2}}-\frac{R^{2} \cdot R_{1}^{2} \cdot q}{R_{1}^{2}-R^{2}} \cdot \frac{1}{r^{2}} \\
\sigma_{\theta}(r)=\frac{q \cdot R_{1}^{2}}{R_{1}^{2}-R^{2}}+\frac{R^{2} \cdot R_{1}^{2} \cdot q}{R_{1}^{2}-R^{2}} \cdot \frac{1}{r^{2}}
\end{array}\right.
$$


Stresses on the inside of the support at $r=R$ are found from the expressions (5).

$$
\left\{\begin{array}{c}
\sigma_{r}(R)=0 \\
\sigma_{\theta}(R)=\frac{2 \cdot q \cdot R_{1}^{2}}{R_{1}^{2}-R^{2}} .
\end{array}\right.
$$

The circumferential deformation of the support for the axisymmetric state in accordance with [10] has the following view:

$$
\varepsilon_{\theta}=\frac{1}{E_{k}} \cdot\left[\sigma_{\theta} \cdot\left(1-v_{k}^{2}\right)-\left(v_{k}+v_{k}^{2}\right) \cdot \sigma_{r}\right]
$$

where, $E_{k}$ - module of linear deformations; $v_{k}$ - Poisson's ratio of the support material.

Substituting expression (6) into (7), taking into account the fact that $v_{k}=0,2 \div 0,3$ and $v_{k}^{2}=0,04 \div 0,09$ are small, we get:

$$
\varepsilon_{\theta}(R)=\frac{1}{E_{k}} \cdot \frac{2 \cdot q \cdot R_{1}}{R_{1}^{2}-R^{2}}=\frac{1}{E_{k}} \cdot \frac{2 \cdot q \cdot R_{1}}{\left(R_{1}-R\right) \cdot\left(R_{1}+R\right)}=\left[R_{1} \approx R\right]=\frac{1}{E_{k}} \cdot \frac{q \cdot R}{h_{k}} .
$$

Vertical displacement (deflection) of the crown is calculated as

$$
u(R)=\varepsilon_{\theta}(R) \cdot R=\frac{1}{E_{k}} \cdot \frac{q \cdot R^{2}}{h_{k}} .
$$

To calculate these displacements, it is necessary to know the reduced height of the support $h_{k}$ and the generalized module of linear deformations of the support material $E_{k}$.

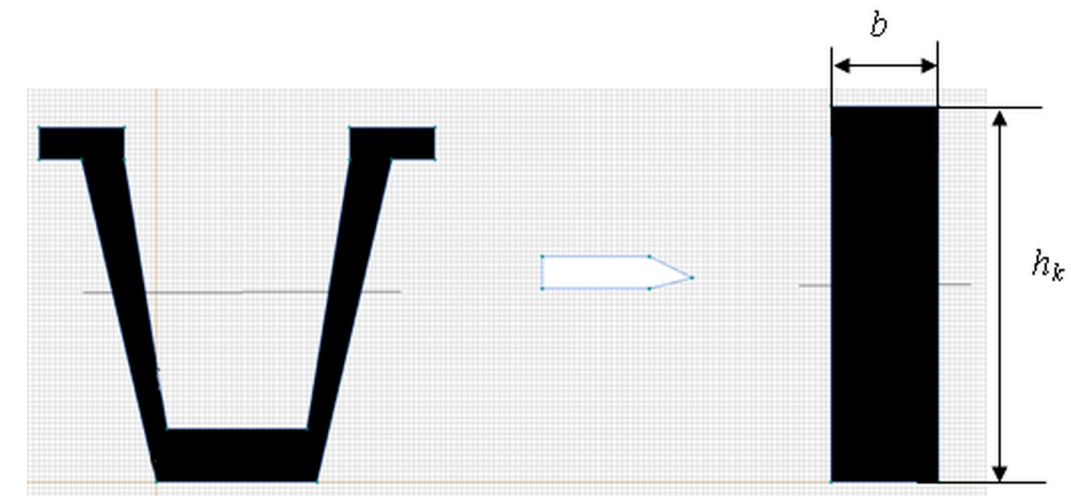

Fig. 2. Support section profile conversion to the equivalent cross-section.

To determine the equivalent cross-section of the support, we replace support section profile SVP with a rectangular with height $h_{k}$ and width $b$ (Fig. 2).

The values of these dimensions are found from the equations of the areas $S$ of the profile and the rectangle and the equation of their inertia moments $I$ with respect to the average section in height. We get the following system of equations: 


$$
\left\{\begin{array}{l}
b \cdot h_{k}=S \\
\frac{b \cdot h_{k}^{3}}{12}=I .
\end{array}\right.
$$

The solution of this system looks as follows:

$$
\left\{\begin{array}{c}
h_{k}=\sqrt{\frac{12 \cdot I}{S}} ; \\
b=\frac{S}{h_{k}} .
\end{array}\right.
$$

The values of the equivalent dimensions of the lining section obtained by formulas (9) are given in the table below.

Table 1. Equivalent dimensions of support cross-section for various SVP profiles [4].

\begin{tabular}{|c|c|c|c|c|}
\hline $\begin{array}{c}\text { Profile } \\
\text { number }\end{array}$ & $\begin{array}{c}\text { Cross-section area } \\
\boldsymbol{S}^{*}, \mathbf{c m}^{\mathbf{2}}\end{array}$ & $\begin{array}{c}\text { Moment of inertia } \\
\boldsymbol{I}^{*} \mathbf{\mathbf { c m } ^ { \mathbf { 4 } }}\end{array}$ & $\begin{array}{c}\text { Equivalent height } \\
\boldsymbol{h} \boldsymbol{k}, \mathbf{c m}\end{array}$ & $\begin{array}{c}\text { Equivalent } \\
\text { width } \boldsymbol{b}, \\
\mathbf{c m}\end{array}$ \\
\hline SVP-17 & 21.73 & 243.4 & 11.59 & 1.87 \\
\hline SVP-19 & 24.44 & 322.8 & 12.59 & 2.22 \\
\hline SVP-22 & 27.91 & 428.6 & 13.57 & 2.06 \\
\hline SVP-27 & 34.37 & 646.1 & 15.02 & 2.29 \\
\hline SVP-33 & 42.53 & 999.5 & 16.79 & 2.53 \\
\hline
\end{tabular}

* Values of areas and moments of inertia for SVP profiles have been taken from ["Profiles for mine roadway support" (pit props)".

The generalized module of linear deformations of the support makes it possible, for calculation purposes, to substitute the structure consisting of the crown, posts and locks with one element - a continuous homogeneous lining with the reduced dimensions and the similar yielding.

To determine the generalized module of linear deformations of the support, the results of bench tests of supports.

The value of the generalized module of linear deformations was determined from the condition of equality of the calculated and full-scale values of the crown deflections with the loss of the frame of its carrying capacity.

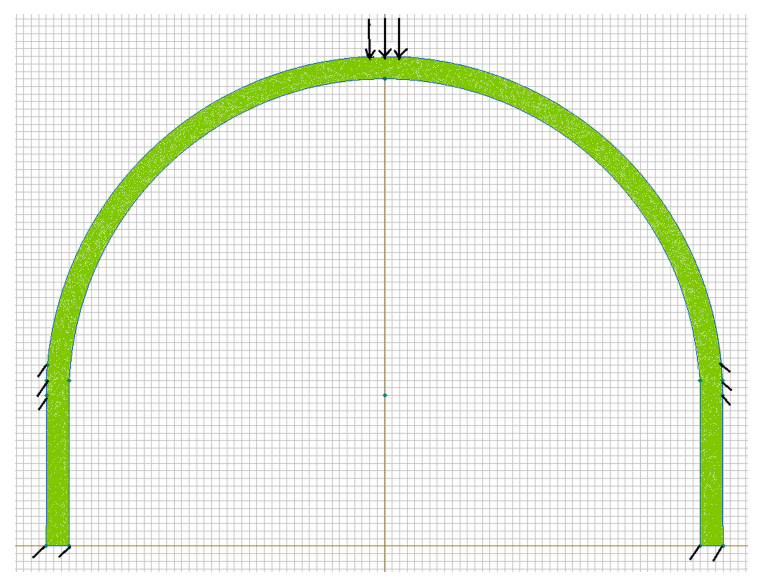

Fig. 3. The bench test scheme in the passive mode. 
The support was calculated as a structure in a two-dimensional stress state. This solution is due to the fact that the horizontal size of the reduced cross-section is an order of magnitude smaller than the vertical size. The design scheme (see Fig. 3) corresponded to the bench test scheme.

The calculation of the stress-strain state of the support was carried out according to the finite element method using the licensed program ELCUT.

The strength of the support was assessed using the Mohr strength criterion:

$$
\sigma_{1}-\frac{\sigma_{t}}{\sigma_{s}} \cdot \sigma_{2}>\sigma_{t}
$$

Where, $\sigma_{1}, \sigma_{2}$ - principal least and maximum strain; $\sigma_{t}, \sigma_{s}-$ strength limits for tensile and compression strength of the support material, $\sigma_{t}=4 \cdot 10^{8} \mathrm{~N} / \mathrm{m}^{2}$, and $\sigma_{t} / \sigma_{S}=0,1$.

Figure 4 shows the results of calculations of the support displacement and the values of the Mohr criterion in the failure limit state.

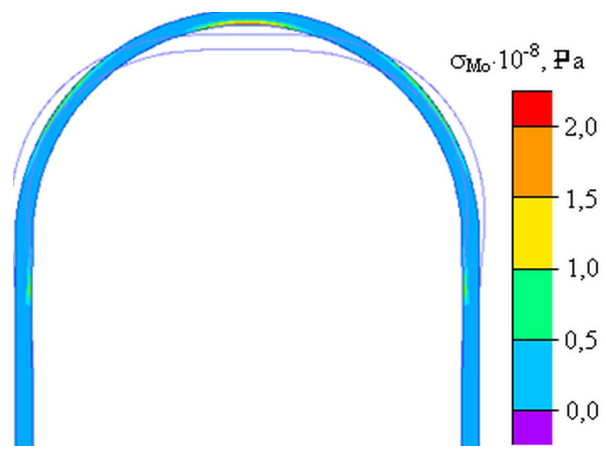

Fig. 4. Displacement of support and distribution of the values of the Mohr criterion in the failure limit state.

The calculated values of the generalized module of linear deformations of the support that satisfy the bench values of the bearing capacity and yielding of the support amount to $2 \cdot 10^{9} \mathrm{~N} / \mathrm{m}^{2}$ and $4 \cdot 10^{9} \mathrm{~N} / \mathrm{m}^{2}$, respectively. The Poisson ratio was set the same as for hotrolled steel profile [16] and amounted to 0.25 .

\section{Conclusions}

1. For an adequate mathematical modeling of the "support - grouting layer - rock mass" system interaction, an axial-symmetric formulation of the problem of support calculation was proposed.

2. The three-piece arch support can be replaced by a ring support with reduced cross dimensions and a generalized module of linear deformations.

3. The values of the module of linear deformations of the ring support, that corresponds in its bearing capacity to the three-piece arch support with profiles SVP-22, SVP-27, are within $(2 \div 4) \cdot 10^{9} \mathrm{~N} / \mathrm{m}^{2}$.

\section{References}

1. G. G. SHtumpf, P.V. Egorov, A.I. Petrov, B.V. Krasil'nikov, Pressure (Nedra, Moscow, 1996) 
2. O. Yakobi, Practice of controlling rock pressure (Nedra, Moscow, 1987)

3. A. E. Klykov, Forum girnikiv, 2, 264 (2013)

4. S.V. Antsiferov, A.S. Sammal, P.V. Deev, IOP Conference Series: Earth and Environmental Science, 50, 012001 (2018)

5. V. Tatsienko, A. Liskovets, M. Sablin, Coal in the 21 st Century: Mining, Intelligent Equipment and Environment Protection conference proceedings, 1, 164 (2018)

6. B.H. Brady, E.T. Brown, Rock Mechanics: For Underground Mining, (Springer Science \& Business Media, New York, 2013)

7. J.C. Jaeger, N.G. Cook, R. Zimmerman, Fundamentals of Rock Mechanics. (John Wiley \& Sons, London, 2009)

8. L. Jing, A Review of Techniques, Journal of Rock Mechanics and Mining Sciences, 40:3, 283 (2003)

9. J.A. Hudson, J.P. Harrison, Engineering Rock Mechanics: An Introduction to the Principles (Elsevier, New York, 2000)

10. S. P. Timoshenko, J. N. Goodier, Theory of elasticity (McGraw-Hill, New York, 1970) 